\title{
The impact of pharmacological and non-pharmacological interventions to improve physical health outcomes in people with schizophrenia: a meta-review of meta-analyses of randomized controlled trials
}

\begin{abstract}
Davy Vancampfort ${ }^{1,2}$, Joseph Firth ${ }^{3-5}$, Christoph U. Correll ${ }^{6-8}$, Marco Solmi ${ }^{9}$, Dan Siskind ${ }^{10,11}$, Marc De Hert ${ }^{2,12}$, Rebekah Carney ${ }^{4}$, Ai Koyanagi ${ }^{13,14}$, André F. Carvalho ${ }^{15,16}$, Fiona Gaughran ${ }^{17,18}$, Brendon Stubbs ${ }^{17,18}$

'KU Leuven Department of Rehabilitation Sciences, Leuven, Belgium; ${ }^{2}$ University Psychiatric Centre KU Leuven, Kortenberg, Belgium; ${ }^{3} \mathrm{NICM}$ Health Research Institute, Western Sydney University, Westmead, Australia; ${ }^{4}$ Division of Psychology and Mental Health, University of Manchester, Manchester, UK; ${ }^{5}$ Centre for Youth Mental Health, University of Melbourne, Melbourne, Australia; ${ }^{6}$ Hofstra Northwell School of Medicine Hempstead, New York, NY, USA; ${ }^{7}$ Department of Psychiatry, Zucker Hillside Hospital, New York, NY, USA; ${ }^{8}$ Department of Child and Adolescent Psychiatry, Charité Universitätsmedizin, Berlin, Germany; ${ }^{9}$ Department of Neurosciences, University of Padua, Padua, Italy; ${ }^{10}$ Metro South Addiction and Mental Health Service, Brisbane, Australia; ' School of Medicine, University of Queensland, Brisbane, Australia; ${ }^{12} \mathrm{KU}$ Leuven Department of Neurosciences, Leuven, Belgium; ' ${ }^{3}$ Research and Development Unit, Parc Sanitari Sant Joan de Déu, Universitat de Barcelona, Fundació Sant Joan de Déu, Barcelona, Spain; ${ }^{14}$ Instituto de Salud Carlos III, Centro de Investigación Biomédica en Red de Salud Mental, CIBERSAM, Madrid, Spain; ${ }^{15}$ Centre for Addiction and Mental Health, Toronto, Ontario, Canada; ${ }^{16}$ Department of Psychiatry, University of Toronto, Toronto, Ontario, Canada; ${ }^{17}$ South London and Maudsley NHS Foundation Trust, London, UK; ${ }^{18}$ Institute of Psychiatry, Psychology and Neuroscience, King's College London, London, UK
\end{abstract}

We summarized and compared meta-analyses of pharmacological and non-pharmacological interventions targeting physical health outcomes among people with schizophrenia spectrum disorders. Major databases were searched until June 1, 2018. Of 3,709 search engine hits, 27 metaanalyses were included, representing 128 meta-analyzed trials and 47,231 study participants. While meta-analyses were generally of adequate or high quality, meta-analyzed studies were less so. The most effective weight reduction interventions were individual lifestyle counseling (standardized mean difference, SMD =-0.98) and exercise interventions (SMD=-0.96), followed by psychoeducation (SMD=-0.77), aripiprazole augmentation (SMD=-0.73), topiramate (SMD=-0.72), d-fenfluramine (SMD=-0.54) and metformin (SMD=-0.53). Regarding waist circumference reduction, aripiprazole augmentation ( $S M D=-1.10)$ and topiramate $(S M D=-0.69)$ demonstrated the best evidence, followed by dietary interventions (SMD=-0.39). Dietary interventions were the only to significantly improve (diastolic) blood pressure (SMD=-0.39). Switching from olanzapine to quetiapine or aripiprazole ( $S M D=-0.71)$ and metformin $(S M D=-0.65)$ demonstrated best efficacy for reducing glucose levels, followed by glucagon-like peptide-1 receptor agonists (SMD=-0.39), dietary interventions (SMD=-0.37) and aripiprazole augmentation (SMD=-0.34), whereas insulin resistance improved the most with metformin ( $S M D=-0.75)$ and rosiglitazone (SMD=-0.44). Topiramate had the greatest efficacy for triglycerides $(S M D=-0.68)$ and low-density lipoprotein $(L D L)$-cholesterol $(S M D=-0.80)$, whereas metformin had the greatest beneficial effects on total cholesterol (SMD=-0.51) and high-density lipoprotein (HDL)-cholesterol (SMD=0.45). Lifestyle interventions yielded small effects for triglycerides, total cholesterol and LDL-cholesterol (SMD=-0.35 to -0.37). Only exercise interventions increased exercise capacity $(S M D=1.81)$. Despite frequent physical comorbidities and premature mortality mainly due to these increased physical health risks, the current evidence for pharmacological and non-pharmacological interventions in people with schizophrenia to prevent and treat these conditions is still limited and more larger trials are urgently needed.

Key words: Schizophrenia, psychosis, physical health, body weight, blood pressure, glucose, insulin, tryglicerides, cholesterol, lifestyle counseling, exercise interventions, dietary interventions, metformin, topiramate, antipsychotic switching

(World Psychiatry 2019;18:53-66)

People with schizophrenia have substantially poorer physical health than the general population ${ }^{1-4}$, which is often attributed to an interaction between social circumstances, lifestyle factors and treatment effects ${ }^{5}$. For instance, behavioral research has demonstrated that people with schizophrenia are less physically active and exhibit more sedentary behavior than the general population ${ }^{6}$, have a higher quantity but lower quality of dietary food intake ${ }^{7}$, and increased adverse health behaviors, such as smoking ${ }^{8}$. Additionally, psychiatric treatment with antipsychotics and other commonly prescribed agents, such as mood stabilizers and antidepressants, further increases the risk of physical health conditions ${ }^{9,10}$. Consequently, people with schizophrenia more frequently have cardio-metabolic diseases ${ }^{11-13}$, respiratory diseases ${ }^{14}$, chronic pain ${ }^{15}$, fractures ${ }^{16}$, and lower physical fitness ${ }^{17,18}$ than the general population.

This increased somatic risk is associated with a lower physical health related quality of life ${ }^{19,20}$, but, despite this increased risk, access to monitoring, physical health care and intervention for those with schizophrenia are suboptimal compared to the general population ${ }^{21,22}$. Resultantly, people with schizophrenia experience a 10-20 year gap in life expectancy, primarily driven by this poorer physical health ${ }^{13,23}$. Furthermore, the physical health inequalities experienced by people with schizophrenia have been observed across the globe ${ }^{24}$ and have not improved over time ${ }^{25}$.

Given this gross inequality, there has been a substantial increase in efforts to improve the physical health of this atrisk population ${ }^{5,26}$. To address the physical health disparity, a number of individual meta-analyses have led to national and international evidence-based recommendations for or against specific pharmacological and non-pharmacological intervention options ${ }^{27-34}$.

Despite this rapid expansion of meta-analytic evidence on interventions for tackling poor physical health in people with 
schizophrenia, no summary of this top-tier of evidence exists, nor is there a direct quantitative comparison of the evidence between all individual and/or combined pharmacological and non-pharmacological strategies. Moreover, the quality of these meta-analyses and the included trials has not been comprehensively evaluated, which is an indispensable step before more rigorous treatment recommendations can confidently be made.

In order to address this gap within the literature, we set out to aggregate the existing top-tier evidence from the most recent/largest published meta-analyses of randomized trials of physical health interventions, in order to determine the comparative quality of evidence and magnitude of efficacy for pharmacological and non-pharmacological interventions targeting physical health outcomes among people with schizophrenia spectrum disorders.

\section{METHODS}

\section{Searches}

Four authors searched independent from each other MEDLINE/PubMed, PsycINFO, EMBASE and the Cochrane databases, from their respective inception dates until June 1, 2018, without language restriction, for meta-analyses of randomized controlled trials in people with schizophrenia spectrum disorders where physical health improvements were the primary outcome.

The search terms included ("meta-analysis" OR "systematic review") AND ("random*" OR "placebo" OR "control*") AND ("schizophrenia" OR "schizoaffective" OR "schizophreniform” OR "psychosis" OR "psychotic" OR "severe mental illness") AND ("physical health" OR "cardio*" OR "metabol*" OR "respir*" OR “*weight" OR "pain" OR "somatic"). We searched the reference lists of all included articles.

\section{Inclusion criteria}

Inclusion criteria were organized in accordance with the population, interventions, comparisons, outcomes and setting/ study design (PICOS) reporting structure (see Table 1).

\section{Data extraction, outcomes, and data synthesis}

Regarding efficacy and adverse drug reactions, we manually extracted effect size data (with $95 \%$ confidence intervals, $\mathrm{CI}$ ) for all relevant outcomes, and the number of participants in the intervention and control arms for each effect size. Specifically, data for effect sizes of continuous outcomes were extracted or recalculated as standardized mean difference (SMD), which expresses the mean difference between the intervention and control groups in standard deviation units, with 95\% CI. Generally, an SMD less than 0.2 is considered negligible, an SMD
Table 1 Application of the PICOS search strategy

Population - People with schizophrenia spectrum disorders, including schizophrenia, schizoaffective or schizophreniform disorder or first episode psychosis, confirmed through validated assessment measures (e.g., DSM, ICD). Studies conducted with a severe mental illness subgroup (e.g., also including bipolar disorder or major depressive disorder) were only included if the schizophrenia spectrum disorder sample was $\geq 70 \%$.

Interventions - We included all pharmacological interventions that had a primary aim to improve physical health outcomes. Non-pharmacological interventions included all educational, psychotherapeutic, social and physical interventions, excluding alternative therapies. Specifically, we included lifestyle interventions (e.g., physical activity, diet, smoking cessation).

Comparisons - All relevant control interventions were included (e.g., placebo, treatment as usual/usual care, waiting list, no treatment).

Outcomes - We considered any physical health outcomes explored, including the following: a) any physical health markers, such as body weight, proportion with overweight or obesity, random or fasting levels of glucose and lipid metabolism parameters, proportion with abnormalities in glucose and lipid metabolism parameters, cardiovascular illness (e.g., myocardial infarction, stroke, transient ischemic attack, pulmonary embolism), respiratory illness (lung cancer, chronic obstructive pulmonary disease); b) parameters of physical fitness (e.g., maximal or peak oxygen uptake, muscle strength); c) any biomarkers investigated (hemoglobin A1c, C-reactive protein or other blood and serum markers); d) any physical health behavior researched (physical activity levels, smoking behavior, diet patterns, attending physical health appointment, attendance rates); e) physical health related quality of life; f) side effects (e.g., adverse drug reactions).

Setting - We considered any setting: hospital (inpatient or outpatient), community, or remote (e.g., using digital technology).

Study design - Meta-analyses informed by a systematic review that included randomized controlled trials (RCTs) were included. A paper was classified as a systematic review and meta-analysis if the following criteria were met: clear inclusion criteria, a systematic search strategy, a screening procedure to identify relevant studies, systematic data extraction and meta-analysis procedures for RCTs. Meta-analyses meeting the inclusion criteria were removed if there was a more recently updated meta-analysis for that same combination strategy and outcome as long as more than $75 \%$ of the meta-analyzed trials overlapped and the pooled sample was larger for that specific intervention and outcome. Conference abstracts were excluded.

between 0.2 and less than 0.5 is small, an SMD between 0.5 and less than 0.8 is medium, and an SMD of at least 0.8 is large ${ }^{35}$. Risk ratios (RRs) were used for categorical outcomes. If odds ratios were present, they were recalculated as RRs. For both types of outcomes, we followed the decisions of the original authors concerning fixed vs. random effects models.

\section{Quality assessment of the meta-analyzed studies}

Included meta-analyses were assessed using "A Measurement Tool to Assess Systematic Reviews" (AMSTAR) (range 0 -11, with a score of 8 or higher indicating high quality) ${ }^{36}$.

While AMSTAR is a reliable and valid tool for measuring the methodological quality of meta-analyses, its score does not capture quality indicators of the meta-analyzed trials, which 
could bias pooled results. For instance, a meta-analysis that meets all methodological quality criteria, but that meta-analyzes potentially biased studies would have a good methodological quality but poor content quality.

Thus, for a more comprehensive assessment of the content validity of included meta-analyses, we used a set of six additional, previously developed quality items, each ranging between 0 and 1 or 2, that capture the content quality of the meta-analyzed trials (AMSTAR-Plus Content, range $0-8$, with a score of 4 or higher indicating high quality) ${ }^{37}$.

\section{Statistical analysis}

We analyzed data as they were directly extracted from the published meta-analyses or, if necessary, after they were converted to standardized outcomes using comprehensive metaanalysis (CMA, version 3) (Biostat). To compare the SMDs of the experimental pharmacological and non-pharmacological interventions vs. the control interventions, we conducted separate random-effects meta-analyses for each variable using CMA.

The AMSTAR and AMSTAR-Plus Content scores and sample size were used where possible in meta-regression analyses, which were done separately for pharmacological and nonpharmacological strategies. Where possible, we also performed meta-regression and subgroup analyses to examine putative factors which may influence effect sizes for each individual physical health outcome, including participant characteristics (e.g., average age, gender distribution) and interventional design (treatment duration in weeks, delivered by mental health vs. physical health staff, clinical setting, percent of sessions attended/adherence, group vs. individual treatment).

Heterogeneity was quantified using the $\mathrm{Q}$ and $\mathrm{I}^{2}$ statistic, with scores of $<25 \%, 25-50 \%$ and $>50 \%$ indicating low, moderate and high heterogeneity, respectively ${ }^{38}$. If it was not possible to extract effect size data for the comparative meta-analysis, we reported individual review level results in a narrative synthesis.

\section{RESULTS}

\section{Systematic search results}

Of 3,709 search engine hits, 27 meta-analyses were includ$\mathrm{ed}^{39-65}$, representing a total of 128 meta-analyzed trials and 47,231 study participants.

There were meta-analytic data for 17 different pharmacological interventions: aripiprazole augmentation ${ }^{43,47,53,55}$, fluoxetine $^{55}$, metformin $^{46,48,49,54,55,61,62}$, nizatidine ${ }^{54,55}$, NMDA receptor antagonists including amantadine and memantine $45,55,57,60$, ranitidine $^{42}$, topiramate ${ }^{39}$, dextroamphetamine ${ }^{64}$, d-fenfluramine ${ }^{64}$, famotidine ${ }^{64}$, metformin in combination with sibutramine ${ }^{64}$, orlistat ${ }^{64}$, rosiglitazone $^{64}$, fluvoxamine ${ }^{64}$, glucagon-like peptide-1 receptor agonists (GLP-1 RAs) ${ }^{40}$, and switching from olanzapine to quetiapine or aripiprazole ${ }^{65}$.
Meta-analytic data were available for six different non-pharmacological interventions: individual lifestyle counseling ${ }^{58,59,63}$, group lifestyle counseling ${ }^{58,59,63}$, cognitive behavioral therapy $^{58,59}$, psychoeducation ${ }^{58}$, exercise ${ }^{50,56,58}$, and dietary interventions $^{44,58}$. One meta-analysis investigated the pooled effect of a combined lifestyle and metformin intervention ${ }^{41}$.

In total, 17 different physical health outcomes were investigated: weight, body mass index, waist circumference, waist to hip ratio, diastolic and systolic blood pressure, fasting glucose, insulin, homeostatic model assessment of insulin resistance, hemoglobin Alc, fasting triglycerides, total cholesterol, highdensity lipoprotein (HDL)-cholesterol, low-density lipoprotein (LDL)-cholesterol, android/gynoid ratio (i.e., percent fat ratio defined as android fat divided by gynoid fat), visceral fat, and functional exercise capacity.

The control interventions included placebo, continued psychotropic treatment, or care as usual for pharmacological trials, and care as usual for non-pharmacological trials.

The number of trials for a specific health outcome ranged from one to 29, with a median of five trials (interquartile range $=$ 5). Trials lasted six to 72 weeks. When reported, the mean age of participants was $34.9 \pm 2.0$ years, and $58.4 \%$ were men.

\section{Quality assessment of the included meta-analyses}

The AMSTAR mean score was $8.8 \pm 1.0$ in the whole sample, $8.9 \pm 0.9$ in the pharmacological interventions, and $8.7 \pm 1.0$ in the non-pharmacological interventions. Twenty-four (89\%) metaanalyzed studies had an AMSTAR score of 8 or higher, but only two $^{42,65}$ (4\%) had the maximum AMSTAR score of 11 . The AMSTAR-Plus Content mean score was $3.4 \pm 1.5$ in the whole sample, $3.2 \pm 1.6$ in the pharmacological interventions, and $3.7 \pm 1.1$ in the non-pharmacological interventions. None had the maximum score of 8.

Only eleven meta-analyses (41\%) were rated as high-quality based on the meta-analyzed studies. Seven of the 27 metaanalyses included only double-blind trials (26\%). In 16 metaanalyses (59\%), the total pooled sample was less than 500 cases, while only five meta-analyses (18\%) had a total sample of more than 1,000 participants. Only two meta-analyses ${ }^{49,53}$ (7\%) had one included trial with at least 200 participants. Finally, following the AMSTAR-Plus Content criteria, a significant heterogeneity was found for 12 meta-analyses (44\%), and $18(67 \%)$ could not disprove the presence of a publication bias.

Further, we examined the relationship between the effect size for both the non-pharmacological and pharmacological interventions versus the control conditions with the quality assessment measures (AMSTAR and AMSTAR-Plus Content). The SMDs for pharmacological and non-pharmacological interventions did not correlate significantly with the methodological quality of the meta-analysis as measured by AMSTAR ( $\mathrm{p}=0.37$ to 0.52 ) nor with the content quality of the metaanalysis as measured by AMSTAR-Plus Content ( $\mathrm{p}=0.17$ to 0.97). 
Table 2 Anthropometric physical health outcomes of pharmacological and non-pharmacological interventions in people with schizophrenia

\begin{tabular}{|c|c|c|c|c|c|c|c|c|c|}
\hline Outcomes & Intervention & SMD & $95 \%$ CI & N. trials & N. participants & AMSTAR & $\begin{array}{c}\text { AMSTAR } \\
\text { Plus Content }\end{array}$ & Effect size & $\begin{array}{l}\text { Between- } \\
\text { group p }\end{array}$ \\
\hline \multirow[t]{24}{*}{ Weight reduction } & & & & & & & & & $<0.001$ \\
\hline & $\begin{array}{l}\text { Individual lifestyle } \\
\text { counseling }\end{array}$ & $-0.98^{* * *}$ & -1.15 to -0.81 & 14 & 411 & 8.3 & 3.7 & Large & \\
\hline & $\begin{array}{l}\text { Exercise } \\
\quad \text { interventions }\end{array}$ & $-0.96^{* * *}$ & -1.27 to -0.66 & 4 & 183 & 8.0 & 2.5 & Large & \\
\hline & Psychoeducation & $-0.77 * * *$ & -0.98 to -0.55 & 8 & 345 & 8.0 & 3.0 & Medium & \\
\hline & $\begin{array}{l}\text { Aripiprazole } \\
\text { augmentation }\end{array}$ & $-0.73^{* * *}$ & -0.97 to -0.48 & 9 & 813 & 8.3 & 3.0 & Medium & \\
\hline & Topiramate & $-0.72 * * *$ & -1.56 to -0.33 & 15 & 783 & 10.0 & 3.0 & Medium & \\
\hline & d-Fenfluramine & $-0.54^{* * *}$ & -1.07 to -0.02 & 1 & 16 & 7.0 & 6.0 & Medium & \\
\hline & Metformin & $-0.53^{* * *}$ & -0.69 to -0.38 & 29 & 1,279 & 8.2 & 3.6 & Medium & \\
\hline & Dietary interventions & $-0.50 * * *$ & -0.66 to -0.34 & 22 & 1,576 & 8.5 & 3.5 & Medium & \\
\hline & $\begin{array}{l}\text { NMDA receptor } \\
\text { antagonists }\end{array}$ & $-0.47^{* * *}$ & -0.62 to -0.32 & 5 & 309 & 8.0 & 4.5 & Small & \\
\hline & $\begin{array}{l}\text { Metformin }+ \text { lifestyle } \\
\text { intervention }\end{array}$ & $-0.44^{* * *}$ & -0.69 to -0.19 & 3 & 122 & 9.0 & 1.0 & Small & \\
\hline & GLP-1 RAs & $-0.44^{* * *}$ & -0.60 to -0.28 & 3 & 168 & 9.0 & 1.0 & Small & \\
\hline & $\begin{array}{l}\text { Group lifestyle } \\
\text { counseling }\end{array}$ & $-0.39^{* * *}$ & -0.54 to -0.23 & 19 & 883 & 8.3 & 3.7 & Small & \\
\hline & Amantadine & $-0.30^{*}$ & -0.57 to -0.03 & 3 & 205 & 8.5 & 3.5 & Small & \\
\hline & $\begin{array}{l}\text { Cognitive behavioral } \\
\text { therapy }\end{array}$ & $-0.37^{*}$ & -0.55 to -0.18 & 11 & 546 & 8.3 & 3.7 & Small & \\
\hline & Nizatidine & $-0.12^{*}$ & -0.24 to 0.00 & 4 & 357 & 8.0 & 3.0 & Negligible & \\
\hline & Ranitidine & -0.24 & -0.67 to 0.20 & 4 & 260 & 11.0 & 1.0 & Non-significant & \\
\hline & $\begin{array}{l}\text { Metformin }+ \\
\text { sibutramine }\end{array}$ & -0.24 & -0.62 to 0.13 & 1 & 28 & 7.0 & 6.0 & Non-significant & \\
\hline & Orlistat & -0.21 & -0.46 to 0.04 & 1 & 63 & 7.0 & 6.0 & Non-significant & \\
\hline & Rosiglitazone & 0.14 & -0.21 to 0.52 & 1 & 29 & 7.0 & 6.0 & Non-significant & \\
\hline & Fluoxetine & 0.14 & -0.09 to 0.36 & 2 & 60 & 7.0 & 3.0 & Non-significant & \\
\hline & Dextroamphetamine & 0.11 & -0.33 to 0.56 & 1 & 20 & 7.0 & 6.0 & Non-significant & \\
\hline & $\begin{array}{c}\text { Switching from } \\
\text { olanzapine to } \\
\text { quetiapine or } \\
\text { aripiprazole }\end{array}$ & -0.11 & -0.23 to 0.03 & 2 & 287 & 11.0 & 3.0 & Non-significant & \\
\hline & Famotidine & -0.02 & -0.48 to 0.43 & 1 & 14 & 7.0 & 6.0 & Non-significant & \\
\hline \multirow{9}{*}{$\begin{array}{l}\text { Body mass index } \\
\text { reduction }\end{array}$} & & & & & & & & & $<0.001$ \\
\hline & Topiramate & $-0.56^{* * *}$ & -1.54 to -0.22 & 11 & 449 & 10.0 & 3.0 & Medium & \\
\hline & $\begin{array}{l}\text { Individual lifestyle } \\
\text { counseling }\end{array}$ & $-0.49^{* * *}$ & -0.77 to -0.22 & 4 & 202 & 8.3 & 3.7 & Small & \\
\hline & GLP-1 RAs & $-0.41^{* * *}$ & -0.57 to -0.26 & 3 & 168 & 9.0 & 1.0 & Small & \\
\hline & $\begin{array}{l}\text { Cognitive } \\
\text { behavioral therapy }\end{array}$ & $-0.34^{*}$ & -0.67 to -0.07 & 6 & 308 & 8.0 & 3.7 & Small & \\
\hline & $\begin{array}{l}\text { Group lifestyle } \\
\text { counseling }\end{array}$ & $-0.28^{*}$ & -0.54 to 0.00 & 4 & 202 & 8.3 & 3.7 & Small & \\
\hline & Metformin & -0.41 & -0.93 to 0.10 & 23 & 1,228 & 9.0 & 3.7 & Non-significant & \\
\hline & Exercise interventions & -0.25 & -0.56 to 0.06 & 8 & 231 & 8.0 & 2.5 & Non-significant & \\
\hline & Ranitidine & -0.23 & -0.44 to 0.00 & 5 & 312 & 11.0 & 1.0 & Non-significant & \\
\hline
\end{tabular}


Table 2 Anthropometric physical health outcomes of pharmacological and non-pharmacological interventions in people with schizophrenia (continued)

\begin{tabular}{|c|c|c|c|c|c|c|c|c|c|}
\hline Outcomes & Intervention & SMD & $95 \%$ CI & N. trials & N. participants & AMSTAR & $\begin{array}{c}\text { AMSTAR } \\
\text { Plus Content }\end{array}$ & Effect size & $\begin{array}{l}\text { Between- } \\
\text { group p }\end{array}$ \\
\hline & $\begin{array}{l}\text { Switching from } \\
\text { olanzapine to } \\
\text { quetiapine }\end{array}$ & -0.12 & -0.29 to 0.05 & 1 & 129 & 11.0 & 3.0 & Non-significant & \\
\hline \multirow{7}{*}{$\begin{array}{l}\text { Waist } \\
\text { circumference } \\
\text { reduction }\end{array}$} & & & & & & & & & $<0.001$ \\
\hline & $\begin{array}{l}\text { Aripiprazole } \\
\text { augmentation }\end{array}$ & $-1.10^{* *}$ & -1.42 to -0.79 & 3 & 174 & 10.0 & 2.0 & Large & \\
\hline & Topiramate & $-0.69^{*}$ & -0.93 to -0.45 & 8 & 310 & 10.0 & 3.0 & Medium & \\
\hline & $\begin{array}{l}\text { Dietary } \\
\quad \text { interventions }\end{array}$ & $-0.39^{* * *}$ & -0.56 to -0.22 & 11 & 858 & 8.5 & 3.5 & Small & \\
\hline & $\begin{array}{l}\text { Lifestyle } \\
\quad \text { interventions }\end{array}$ & $-0.37^{* *}$ & -0.60 to -0.13 & 10 & 705 & 8.0 & 5.0 & Small & \\
\hline & GLP-1 RAs & $-0.34^{* * *}$ & -0.50 to -0.18 & 3 & 167 & 9.0 & 1.0 & Small & \\
\hline & Metformin & -0.01 & -0.68 to 0.65 & 12 & 721 & 9.0 & 6.0 & Non-significant & \\
\hline \multirow{4}{*}{$\begin{array}{l}\text { Waist to hip ratio } \\
\text { reduction }\end{array}$} & & & & & & & & & 0.07 \\
\hline & Topiramate & $-0.69^{* *}$ & -0.90 to 0.27 & 5 & 123 & 10.0 & 3.0 & Medium & \\
\hline & Metformin & -0.32 & -1.15 to 0.51 & 3 & 133 & 8.0 & 5.0 & Non-significant & \\
\hline & GLP-1 RAs & 0.03 & -0.13 to 0.18 & 3 & 163 & 9.0 & 1.0 & Non-significant & \\
\hline \multicolumn{10}{|l|}{$\begin{array}{l}\text { Android/gynoid } \\
\text { ratio }\end{array}$} \\
\hline & GLP-1 RAs & -0.03 & -0.20 to 0.13 & 3 & 131 & 9.0 & 1.0 & Non-significant & \\
\hline \multicolumn{10}{|l|}{$\begin{array}{l}\text { Visceral fat } \\
\text { reduction }\end{array}$} \\
\hline & GLP-1 RAs & $-0.37^{*}$ & -0.46 to -0.06 & 3 & 97 & 9.0 & 1.0 & Small & \\
\hline
\end{tabular}

${ }^{*} \mathrm{p}<0.05,{ }^{* *} \mathrm{p}<0.01,{ }^{* * *} \mathrm{p}<0.001$

SMD - standardized mean difference, GLP-1 RAs - glucagon-like peptide-1 receptor agonists, AMSTAR - A Measurement Tool to Assess Systematic Reviews mean scores

\section{Physical health outcomes of pharmacological and non-pharmacological interventions}

An overview of the different physical health outcomes of pharmacological and non-pharmacological interventions in people with schizophrenia based on the SMDs and the quality of the meta-analyzed studies as assessed by the AMSTAR and AMSTARPlus scores is presented in Table 2 (anthropometric results) and Table 3 (blood pressure, metabolic and exercise capacity results).

\section{Body weight}

\section{Non-pharmacological interventions}

Six meta-analyses investigated non-pharmacological interventions for body weight (78 trials, $\mathrm{N}=3,944)$. The mean AMSTAR score was $8.2 \pm 0.2$ and the mean AMSTAR-Plus Content was $3.3 \pm 0.4$.
Individual lifestyle counseling was the most effective intervention ( $\mathrm{SMD}=-0.98,95 \% \mathrm{CI}:-1.15$ to $-0.81, \mathrm{p}<0.001 ; 14$ trials, $\mathrm{N}=411, \mathrm{I}^{2}=0 \%, \mathrm{Q}=0.5$, i.e., large effect), followed by exercise interventions alone (SMD $=-0.96,95 \% \mathrm{CI}:-1.27$ to $-0.66, \mathrm{p}<0.001$; 4 trials, $\mathrm{N}=183, \mathrm{I}^{2}=0, \mathrm{Q}=0$, i.e., large effect).

Psychoeducation interventions focusing on promoting a healthy lifestyle showed a medium effect (SMD $=-0.77,95 \%$ CI: -0.98 to $-0.55, \mathrm{p}<0.001 ; 8$ trials, $\mathrm{N}=345, \mathrm{I}^{2}=0, \mathrm{Q}=0$ ). This was also the case for dietary interventions alone (SMD $=-0.50,95 \%$ CI: -0.66 to $-0.34, \mathrm{p}<0.001 ; 22$ trials, $\mathrm{N}=1,576, \mathrm{I}^{2}=94 \%, \mathrm{Q}=15.8$ ).

A small effect was observed for cognitive behavioral therapy focusing on promoting a healthy lifestyle (SMD $=-0.37,95 \%$ CI: -0.55 to $-0.18, \mathrm{p}=0.022 ; 11$ trials, $\mathrm{N}=546, \mathrm{I}^{2}=0 \%, \mathrm{Q}=0.2$ ) and group lifestyle counseling (SMD $=-0.39,95 \% \mathrm{CI}$ : -0.54 to -0.23 , $\mathrm{p}<0.001 ; 19$ trials, $\mathrm{N}=883, \mathrm{I}^{2}=28 \%, \mathrm{Q}=2.8$ ).

With regards to prevention of weight increase, multidisciplinary lifestyle/behavioral counseling showed a medium effect (SMD $=-0.69,95 \%$ CI: -0.84 to $-0.54, \mathrm{p}<0.001 ; 14$ trials, $\mathrm{N}=694$, $\left.\mathrm{I}^{2}=0 \%, \mathrm{Q}=1.4\right)$. 
Table 3 Blood pressure, metabolic and exercise capacity physical health outcomes of pharmacological and non-pharmacological interventions in people with schizophrenia

\begin{tabular}{|c|c|c|c|c|c|c|c|c|c|}
\hline Outcomes & Intervention & SMD & $95 \%$ CI & N. trials & N. participants & AMSTAR & $\begin{array}{c}\text { AMSTAR } \\
\text { Plus Content }\end{array}$ & Effect size & $\begin{array}{l}\text { Between- } \\
\text { group p }\end{array}$ \\
\hline \multirow{5}{*}{$\begin{array}{l}\text { Systolic blood } \\
\text { pressure } \\
\text { reduction }\end{array}$} & & & & & & & & & 0.14 \\
\hline & Metformin & -0.24 & -0.53 to 0.05 & 3 & 176 & 9.0 & 4.0 & Non-significant & \\
\hline & Lifestyle interventions & -0.22 & -0.49 to 0.05 & 7 & 615 & 8.0 & 5.0 & Non-significant & \\
\hline & GLP-1 RAs & -0.09 & -0.24 to 0.06 & 3 & 160 & 9.0 & 1.0 & Non-significant & \\
\hline & Dietary interventions & 0.05 & -0.18 to 0.28 & 7 & 655 & 8.5 & 3.5 & Non-significant & \\
\hline \multirow{5}{*}{$\begin{array}{l}\text { Diastolic blood } \\
\text { pressure } \\
\text { reduction }\end{array}$} & & & & & & & & & $<0.001$ \\
\hline & Dietary interventions & $-0.39 * *$ & -0.56 to -0.22 & 6 & 654 & 8.5 & 3.5 & Small & \\
\hline & Metformin & -0.24 & -0.53 to 0.05 & 3 & 176 & 9.0 & 4.0 & Non-significant & \\
\hline & GLP-1 RAs & -0.12 & -0.28 to 0.03 & 3 & 160 & 9.0 & 1.0 & Non-significant & \\
\hline & Lifestyle interventions & -0.08 & -0.57 to 0.41 & 3 & 171 & 8.0 & 5.0 & Non-significant & \\
\hline \multirow{8}{*}{$\begin{array}{l}\text { Glucose level } \\
\text { reduction }\end{array}$} & & & & & & & & & $<0.001$ \\
\hline & $\begin{array}{c}\text { Switching from } \\
\text { olanzapine to } \\
\text { quetiapine or } \\
\text { aripiprazole }\end{array}$ & $-0.71^{* * *}$ & -0.85 to -0.58 & 2 & 280 & 11.0 & 3.0 & Medium & \\
\hline & Metformin & $-0.65^{* * *}$ & -0.94 to -0.35 & 17 & 1,281 & 9.6 & 3.7 & Medium & \\
\hline & GLP-1 RAs & $-0.39 * * *$ & -0.54 to -0.23 & 3 & 166 & 9.0 & 1.0 & Small & \\
\hline & Dietary interventions & $-0.37^{*}$ & -0.69 to -0.05 & 6 & 422 & 8.5 & 3.5 & Small & \\
\hline & $\begin{array}{l}\text { Aripiprazole } \\
\text { augmentation }\end{array}$ & $-0.34^{* * *}$ & -0.47 to -0.20 & 10 & 710 & 9.3 & 3.5 & Small & \\
\hline & Topiramate & -0.43 & -1.00 to 0.14 & 6 & 369 & 10.0 & 3.0 & Non-significant & \\
\hline & Lifestyle interventions & -0.27 & -0.59 to 0.05 & 8 & 688 & 8.0 & 5.0 & Non-significant & \\
\hline \multirow{5}{*}{$\begin{array}{l}\text { Insulin level } \\
\text { reduction }\end{array}$} & & & & & & & & & $<0.001$ \\
\hline & Rosiglitazone & $-0.42^{*}$ & -0.80 to 0.00 & 1 & 29 & 7.0 & 6.0 & Small & \\
\hline & Lifestyle interventions & $-0.28^{*}$ & -0.55 to 0.00 & 6 & 481 & 8.0 & 5.0 & Small & \\
\hline & Metformin & -0.37 & -0.81 to 0.07 & 15 & 1,007 & 9.5 & 4.5 & Non-significant & \\
\hline & Dietary interventions & -0.19 & -0.42 to 0.04 & 11 & 787 & 8.5 & 3.5 & Non-significant & \\
\hline \multirow{4}{*}{$\begin{array}{l}\text { HOMA-IR } \\
\text { improvement }\end{array}$} & & & & & & & & & $<0.001$ \\
\hline & Metformin & $-0.75^{* * *}$ & -1.10 to -0.40 & 11 & 680 & 9.0 & 6.0 & Medium & \\
\hline & Rosiglitazone & $-0.44^{*}$ & -0.82 to -0.06 & 1 & 29 & 7.0 & 6.0 & Small & \\
\hline & GLP-1 RAs & -0.08 & -0.23 to 0.08 & 3 & 163 & 9.0 & 1.0 & Non-significant & \\
\hline \multicolumn{10}{|l|}{$\mathrm{HbA1c}$ reduction } \\
\hline & Metformin & $-0.38^{*}$ & -0.69 to -0.07 & 4 & 383 & 9.0 & 6.0 & Small & \\
\hline & GLP-1 RAs & $-0.38^{*}$ & -0.53 to -0.22 & 3 & 166 & 9.0 & 1.0 & Small & \\
\hline \multirow{4}{*}{$\begin{array}{l}\text { Triglycerides } \\
\text { reduction }\end{array}$} & & & & & & & & & $<0.001$ \\
\hline & Topiramate & $-0.68^{*}$ & -1.23 to -0.13 & 5 & 268 & 10.0 & 3.0 & Medium & \\
\hline & Lifestyle interventions & $-0.37 * * *$ & -0.54 to -0.20 & 8 & 659 & 8.0 & 5.0 & Small & \\
\hline & Metformin & $-0.28^{* * *}$ & -0.45 to -0.11 & 11 & 856 & 9.0 & 4.0 & Small & \\
\hline
\end{tabular}


Table 3 Blood pressure, metabolic and exercise capacity physical health outcomes of pharmacological and non-pharmacological interventions in people with schizophrenia (continued)

\begin{tabular}{|c|c|c|c|c|c|c|c|c|c|}
\hline Outcomes & Intervention & SMD & $95 \% \mathrm{CI}$ & N. trials & N. participants & AMSTAR & $\begin{array}{c}\text { AMSTAR } \\
\text { Plus Content }\end{array}$ & Effect size & $\begin{array}{l}\text { Between- } \\
\text { group p }\end{array}$ \\
\hline & $\begin{array}{l}\text { Aripiprazole } \\
\text { augmentation }\end{array}$ & $-0.17^{* *}$ & -0.30 to -0.04 & 9 & 631 & 9.5 & 3.5 & Negligible & \\
\hline & GLP-1 RAs & $-0.15^{*}$ & -0.31 to -0.02 & 3 & 166 & 9.0 & 1.0 & Negligible & \\
\hline & Dietary interventions & -0.15 & -0.30 to 0.00 & 7 & 611 & 8.5 & 3.5 & Non-significant & \\
\hline \multirow{6}{*}{$\begin{array}{l}\text { Total cholesterol } \\
\text { reduction }\end{array}$} & & & & & & & & & 0.02 \\
\hline & Metformin & $-0.51^{* * *}$ & -0.81 to -0.20 & 8 & 628 & 9.0 & 6.0 & Medium & \\
\hline & Lifestyle interventions & $-0.35^{* *}$ & -0.54 to -0.16 & 7 & 590 & 8.0 & 5.0 & Small & \\
\hline & $\begin{array}{l}\text { Aripiprazole } \\
\text { augmentation }\end{array}$ & $-0.32^{* * *}$ & -0.47 to -0.17 & 10 & 692 & 9.3 & 3.5 & Small & \\
\hline & Topiramate & -0.75 & -1.57 to 0.07 & 3 & 187 & 10.0 & 3.0 & Non-significant & \\
\hline & Dietary interventions & -0.13 & -0.29 to 0.03 & 7 & 621 & 8.5 & 3.5 & Non-significant & \\
\hline \multirow{7}{*}{$\begin{array}{l}\text { HDL-cholesterol } \\
\text { elevation }\end{array}$} & & & & & & & & & 0.007 \\
\hline & Metformin & $0.45^{*}$ & 0.00 to 0.90 & 7 & 542 & 9.0 & 6.0 & Small & \\
\hline & Lifestyle interventions & 0.28 & -0.16 to 0.72 & 8 & 627 & 8.0 & 5.0 & Non-significant & \\
\hline & GLP-1 RAs & -0.04 & -0.19 to 0.11 & 3 & 166 & 9.0 & 1.0 & Non-significant & \\
\hline & Topiramate & -0.07 & -0.57 to 0.43 & 4 & 247 & 10.0 & 3.0 & Non-significant & \\
\hline & Dietary interventions & -0.09 & -0.24 to 0.06 & 7 & 547 & 8.5 & 3.5 & Non-significant & \\
\hline & $\begin{array}{l}\text { Aripiprazole } \\
\text { augmentation }\end{array}$ & -0.27 & -0.44 to 0.01 & 8 & 544 & 9.3 & 3.5 & Non-significant & \\
\hline \multirow{6}{*}{$\begin{array}{l}\text { LDL-cholesterol } \\
\text { reduction }\end{array}$} & & & & & & & & & $<0.001$ \\
\hline & Topiramate & $-0.80 * * *$ & -1.06 to -0.53 & 4 & 247 & 10.0 & 3.0 & Large & \\
\hline & Lifestyle interventions & $-0.36^{* *}$ & -0.60 to -0.12 & 5 & 590 & 8.0 & 5.0 & Small & \\
\hline & GLP-1 RAs & $-0.17^{*}$ & -0.32 to -0.02 & 3 & 162 & 9.0 & 1.0 & Negligible & \\
\hline & Metformin & -0.11 & -0.31 to 0.09 & 5 & 433 & 9.0 & 6.0 & Non-significant & \\
\hline & $\begin{array}{l}\text { Aripiprazole } \\
\text { augmentation }\end{array}$ & -0.01 & -0.18 to 0.15 & 8 & 540 & 9.3 & 3.5 & Non-significant & \\
\hline $\begin{array}{l}\text { Functional } \\
\text { exercise capacity }\end{array}$ & Exercise interventions & $1.81^{* *}$ & 0.59 to 3.03 & 1 & 13 & 8.0 & 2.0 & Large & \\
\hline
\end{tabular}

${ }^{*} \mathrm{p}<0.05,{ }^{* *} \mathrm{p}<0.01,{ }^{* * *} \mathrm{p}<0.001$

SMD - standardized mean difference, GLP-1 RAs - glucagon-like peptide-1 receptor agonists, AMSTAR - A Measurement Tool to Assess Systematic Reviews mean scores, HbA1c - hemoglobin Alc, HOMA-IR - homeostatic model assessment of insulin resistance, HDL - high-density lipoprotein, LDL - low-density lipoprotein

\section{Pharmacological interventions}

Altogether, 14 meta-analyses investigated pharmacological interventions for body weight ( 82 trials, $\mathrm{N}=4,691$ ). The mean AMSTAR score was $8.1 \pm 1.3$ and the mean AMSTAR-Plus Content was $4.0 \pm 1.7$.

A medium effect size was observed for aripiprazole augmentation (SMD $=-0.73,95 \% \mathrm{CI}:-0.97$ to $-0.48, \mathrm{p}<0.001 ; 9$ trials, $\mathrm{N}=813, \mathrm{I}^{2}=68 \%, \mathrm{Q}=6.2$ ), topiramate (SMD $=-0.72,95 \% \mathrm{CI}$ : -1.56 to $-0.33, \mathrm{p}<0.001 ; 15$ trials, $\mathrm{N}=783, \mathrm{I}^{2}=92.7 \%, \mathrm{Q}=13.7$ ), d-fenfluramine (SMD $=-0.54,95 \% \mathrm{CI}:-1.07$ to -0.02 , $\mathrm{p}<0.001$; one trial, $\mathrm{N}=16)$ and metformin (SMD $=-0.53,95 \% \mathrm{CI}:-0.69$ to $-0.38, \mathrm{p}<0.001 ; 29$ trials, $\left.\mathrm{N}=1,279, \mathrm{I}^{2}=39.4 \%, \mathrm{Q}=1.6\right)$.

A small significant effect was shown by NMDA receptor antagonists (SMD $=-0.47,95 \% \mathrm{CI}:-0.62$ to $-0.32, \mathrm{p}<0.001 ; 5$ trials, $\mathrm{N}=309, \mathrm{I}^{2}=0 \%, \mathrm{Q}=0.1$ ), GLP-1 RAs (SMD $=-0.44,95 \% \mathrm{CI}:-0.60$ to $-0.28, \mathrm{p}<0.001 ; 3$ trials, $\left.\mathrm{N}=168, \mathrm{I}^{2}=0 \%, \mathrm{Q}=0.1\right)$ and amantadine ( $\mathrm{SMD}=-0.30,95 \% \mathrm{CI}:-0.57$ to $-0.03, \mathrm{p}=0.03 ; 3$ trials, $\mathrm{N}=205$, $\mathrm{I}^{2}=0 \%, \mathrm{Q}=0$ ). Nizatidine showed a negligible effect (SMD $=-0.12$, 95\% CI: -0.24 to $0.00, \mathrm{p}=0.02$; 4 trials, $\mathrm{N}=357, \mathrm{I}^{2}=0 \%, \mathrm{Q}=0.4$ ).

No significant weight loss compared to the control condition was observed for fluoxetine (SMD $=0.14,95 \%$ CI: -0.09 
to $0.36, \mathrm{p}=0.22 ; 2$ trials, $\mathrm{N}=60, \mathrm{I}^{2}=0 \%, \mathrm{Q}=0$ ), dextroamphetamine (SMD $=0.11,95 \% \mathrm{CI}$ : -0.33 to $0.56, \mathrm{p}=0.60$; one trial, $\mathrm{N}=20$ ), ranitidine ( $\mathrm{SMD}=-0.24,95 \% \mathrm{CI}:-0.67$ to $0.20, \mathrm{p}=0.05$; 4 trials, $\mathrm{N}=260$ ), famotidine ( $\mathrm{SMD}=-0.02,95 \% \mathrm{CI}$ : -0.48 to 0.43 , $\mathrm{p}=0.91$; one trial, $\mathrm{N}=14$ ), the combination of metformin with sibutramine ( $\mathrm{SMD}=-0.24,95 \% \mathrm{CI}$ : -0.62 to $0.13, \mathrm{p}=0.19$; one trial, $\mathrm{N}=28$ ), orlistat (SMD $=-0.21,95 \% \mathrm{CI}:-0.46$ to 0.04 , $\mathrm{p}=0.09$; one trial, $\mathrm{N}=63$ ) and rosiglitazone ( $\mathrm{SMD}=0.14,95 \% \mathrm{CI}:-0.21$ to 0.52 , $\mathrm{p}=0.19$; one trial, $\mathrm{N}=29$ ). Switching from olanzapine to quetiapine or aripiprazole fell also short of statistical significance ( $\mathrm{SMD}=-0.11,95 \% \mathrm{CI}:-0.23$ to $0.03, \mathrm{p}=0.06 ; 2$ trials, $\mathrm{N}=287$ ).

\section{Combined pharmacological and non-pharmacological interventions}

The combination of metformin with a lifestyle intervention was explored in one meta-analysis and demonstrated a small effect (SMD $=-0.44,95 \% \mathrm{CI}$ : -0.69 to -0.19 , $\mathrm{p}<0.001 ; 3$ trials, $\left.\mathrm{N}=122, \mathrm{I}^{2}=0, \mathrm{Q}=0\right)$.

\section{Body mass index}

\section{Non-pharmacological interventions}

Four meta-analyses investigated non-pharmacological interventions for body mass index (42 trials, $\mathrm{N}=2,157$ ). The mean AMSTAR score was $9.7 \pm 0.8$ and the mean AMSTAR-Plus Content was $2.2 \pm 1.2$.

A small effect was observed for group lifestyle counseling ( $\mathrm{SMD}=-0.28,95 \% \mathrm{CI}:-0.54$ to $0.00, \mathrm{p}=0.04 ; 4$ trials, $\mathrm{N}=202$, $\left.\mathrm{I}^{2}=0 \%, \mathrm{Q}=0\right)$, individual lifestyle counseling ( $\mathrm{SMD}=-0.49,95 \%$ CI: -0.77 to $-0.22, \mathrm{p}<0.001 ; 4$ trials, $\mathrm{N}=202, \mathrm{I}^{2}=0 \%, \mathrm{Q}=0$ ), and cognitive behavioral therapy focusing on promoting healthy lifestyles (SMD $=-0.34,95 \% \mathrm{CI}:-0.67$ to $-0.07, \mathrm{p}=0.02 ; 6$ trials, $\mathrm{N}=308, \mathrm{I}^{2}=0 \%, \mathrm{Q}=0.2$ ).

No significant reduction in body mass index compared to the control condition was observed for exercise interventions $\left(\mathrm{SMD}=-0.25,95 \% \mathrm{CI}:-0.56\right.$ to $0.06, \mathrm{p}=0.11 ; 8$ trials, $\mathrm{N}=231, \mathrm{I}^{2}$ $=0 \%, \mathrm{Q}=0.4)$.

\section{Pharmacological interventions}

Six meta-analyses investigated pharmacological interventions for body mass index ( 81 trials, $N=4,533$ ). The mean AMSTAR score was $8.1 \pm 1.3$ and the mean AMSTAR-Plus Content was $4.0 \pm 1.9$.

Topiramate (SMD $=-0.56,95 \% \mathrm{CI}:-1.54$ to $-0.22, \mathrm{p}<0.001 ; 11$ trials, $\mathrm{N}=449, \mathrm{I}^{2}=0 \%, \mathrm{Q}=0$ ) had a medium reducing effect, while GLP-1 RAs demonstrated a small effect (SMD $=-0.41,95 \% \mathrm{CI}$ : -0.57 to $-0.26, \mathrm{p}<0.001 ; 3$ trials, $\mathrm{N}=168, \mathrm{I}^{2}=0 \%, \mathrm{Q}=0$ ).

No reduction of body mass index was observed with metformin ( $\mathrm{SMD}=-0.41,95 \% \mathrm{CI}:-0.93$ to $0.10, \mathrm{p}=0.10 ; 23$ trials, $\mathrm{N}=1,228, \mathrm{I}^{2}=90 \%, \mathrm{Q}=20.0$ ), ranitidine ( $\mathrm{SMD}=-0.23,95 \% \mathrm{CI}$ : -0.44 to $0.00, \mathrm{p}=0.55 ; 5$ trials, $\mathrm{N}=312, \mathrm{I}^{2}=0 \%, \mathrm{Q}=0$ ), and switch- ing from olanzapine to quetiapine (SMD=-0.12, 95\% CI: -0.29 to $0.05, \mathrm{p}=0.16$; one trial, $\mathrm{N}=129$ ).

\section{Waist circumference}

\section{Non-pharmacological interventions}

Three meta-analyses investigated non-pharmacological interventions for waist circumference reduction (24 trials, $\mathrm{N}=$ $1,709)$. The mean AMSTAR score was $9.3 \pm 0.5$ and the mean AMSTAR-Plus Content was 4.0 \pm 0.8 .

A small waist circumference reduction effect compared to care as usual was observed for dietary interventions (SMD $=-0.39$, 95\% CI: -0.56 to -0.22 , $\mathrm{p}<0.001 ; 11$ trials, $\left.\mathrm{N}=858, \mathrm{I}^{2}=0 \%, \mathrm{Q}=0\right)$ and multidisciplinary lifestyle/behavioral interventions (SMD $=-0.37$, 95\% CI: -0.60 to $-0.13, \mathrm{p}=0.002 ; 10$ trials, $\mathrm{N}=705, \mathrm{I}^{2}=0 \%, \mathrm{Q}=0$ ).

\section{Pharmacological interventions}

Seven meta-analyses investigated pharmacological interventions for waist circumference reduction (32 trials, $\mathrm{N}=1,755$ ). The mean AMSTAR score was $9.3 \pm 0.7$ and the mean AMSTARPlus Content was 2.8 \pm 1.6 .

Compared with the control condition, the most effective intervention was aripiprazole augmentation $(\mathrm{SMD}=-1.10,95 \%$ CI: -1.42 to $-0.79, \mathrm{p}=0.001 ; 3$ trials, $\mathrm{N}=174, \mathrm{I}^{2}=0 \%, \mathrm{Q}=0$, i.e., large effect).

Topiramate had a medium effect $(\mathrm{SMD}=-0.69,95 \% \mathrm{CI}:-0.93$ to $-0.45, \mathrm{p}<0.05 ; 8$ trials, $\left.\mathrm{N}=310, \mathrm{I}^{2}=0 \%, \mathrm{Q}=0\right)$. GLP-1 RAs had a small effect (SMD $=-0.34,95 \% \mathrm{CI}:-0.50$ to $-0.18, \mathrm{p}<0.001 ; 3$ trials, $\mathrm{N}=167, \mathrm{I}^{2}=0 \%, \mathrm{Q}=0$ ). No significant waist circumference reduction compared to the placebo control condition was observed for metformin ( $\mathrm{SMD}=-0.01,95 \% \mathrm{CI}$ : -0.68 to $0.65, \mathrm{p}=0.97$; 12 trials, $\mathrm{N}=721, \mathrm{I}^{2}=82 \%, \mathrm{Q}=17.1$ ).

\section{Waist to hip ratio}

Three meta-analyses investigated the effects of pharmacological interventions on the waist to hip ratio ( 11 trials, $\mathrm{N}=419$ ). The mean AMSTAR score was $9.3 \pm 0.5$ and the mean AMSTARPlus Content was $3.3 \pm 2.0$.

A small waist to hip ratio reduction compared to the control condition was observed for topiramate (SMD $=-0.69,95 \% \mathrm{CI}$ : -0.90 to $0.27, \mathrm{p}=0.009 ; 5$ trials, $\mathrm{N}=123, \mathrm{I}^{2}=0 \%, \mathrm{Q}=0$ ) and metformin (SMD $=-0.32,95 \% \mathrm{CI}:-1.15$ to $0.51, \mathrm{p}=0.29 ; 3$ trials, $\mathrm{N}=133, \mathrm{I}^{2}=0 \%$, $\mathrm{Q}=0$ ). GLP-1 RAs demonstrated no significant effect ( $\mathrm{SMD}=0.03$, 95\% CI: -0.13 to $0.18, \mathrm{p}=0.39 ; 3$ trials, $\mathrm{N}=163, \mathrm{I}^{2}=0 \%, \mathrm{Q}=0$ ).

\section{Android/gynoid ratio}

Based on data from one meta-analysis, GLP-1 RAs did not outperform the control condition concerning the effect on an- 
droid/gynoid ratio (SMD=-0.03, 95\% CI: -0.20 to $0.13, \mathrm{p}=0.46$; 3 trials, $\left.\mathrm{N}=131, \mathrm{I}^{2}=0 \%, \mathrm{Q}=0\right)$.

\section{Visceral fat}

Based on data from one meta-analysis, GLP-1 RAs had a small effect in reducing visceral fat (SMD $=-0.37,95 \% \mathrm{CI}:-0.46$ to $-0.06, \mathrm{p}=0.02 ; 3$ trials, $\mathrm{N}=97, \mathrm{I}^{2}=0 \%, \mathrm{Q}=0$ ).

\section{Blood pressure}

\section{Non-pharmacological interventions}

Four meta-analyses investigated non-pharmacological interventions for blood pressure reduction (23 trials, $\mathrm{N}=2,095$ ). The mean AMSTAR score was $9.0 \pm 0.0$ and the mean AMSTARPlus Content was 4.5 \pm 0.5 .

When looking at dietary interventions alone, a small reduction in diastolic blood pressure versus care as usual was observed (SMD $=-0.39,95 \% \mathrm{CI}:-0.56$ to $-0.22, \mathrm{p}<0.01 ; 6$ trials, $\mathrm{N}=654$, $\mathrm{I}^{2}=0, \mathrm{Q}=0$ ).

Compared to care as usual, no significant reduction in systolic (SMD $=-0.22,95 \% \mathrm{CI}:-0.49$ to $0.05, \mathrm{p}=0.11 ; 7$ trials, $\mathrm{N}=615$, $\left.\mathrm{I}^{2}=0, \mathrm{Q}=0\right)$ and diastolic blood pressure ( $\mathrm{SMD}=-0.08,95 \% \mathrm{CI}$ : -0.57 to $0.41, \mathrm{p}=0.74 ; 3$ trials, $\mathrm{N}=171, \mathrm{I}^{2}=0, \mathrm{Q}=0$ ) was observed for multidisciplinary lifestyle/behavioral interventions.

\section{Pharmacological interventions}

Two meta-analyses investigated pharmacological interventions for blood pressure ( 6 trials, $\mathrm{N}=336$ ). The mean AMSTAR score was 9.5 \pm 0.5 and the mean AMSTAR-Plus Content was $1.5 \pm 0.5$.

Compared to the placebo condition, no significant reduction in systolic and diastolic blood pressure was observed for metformin and GLP-1 RAs.

\section{Glucose}

\section{Non-pharmacological interventions}

Three meta-analyses investigated non-pharmacological interventions on fasting glucose levels (16 trials, $N=1,256)$. The mean AMSTAR score was $9.3 \pm 0.5$ and the mean AMSTAR-Plus Content was $4.0 \pm 0.8$.

Dietary interventions showed a small glucose level reducing effect ( $\mathrm{SMD}=-0.37,95 \% \mathrm{CI}$ : -0.69 to $-0.05, \mathrm{p}=0.03 ; 6$ trials, $\mathrm{N}=422, \mathrm{I}^{2}=0 \%, \mathrm{Q}=0$ ).

No significant reduction in glucose levels compared to care as usual was observed for multidisciplinary lifestyle/behavioral interventions (SMD $=-0.27,95 \% \mathrm{CI}$ : -0.59 to $0.05, \mathrm{p}=0.10$; 8 trials, $\mathrm{N}=688, \mathrm{I}^{2}=0 \%, \mathrm{Q}=0$ ).

\section{Pharmacological interventions}

Seven meta-analyses investigated the effect of pharmacological interventions on fasting glucose levels (54 trials, $N=3,617$ ). The mean AMSTAR score was $9.6 \pm 0.6$ and the mean AMSTARPlus Content was 3.1 \pm 1.9 .

A medium fasting glucose level lowering effect was found for switching olanzapine to quetiapine or aripiprazole (SMD= $-0.71,95 \%$ CI: -0.85 to $-0.58, \mathrm{p}<0.001 ; 2$ trials, $\mathrm{N}=280$ ), and for metformin (SMD $=-0.65,95 \% \mathrm{CI}:-0.94$ to -0.35 , $\mathrm{p}<0.001 ; 17$ trials, $\mathrm{N}=1281, \mathrm{I}^{2}=0 \%, \mathrm{Q}=0$ ).

The effect was small for aripiprazole augmentation (SMD= $-0.34,95 \%$ CI: -0.47 to $-0.21, \mathrm{p}<0.001 ; 10$ trials, $\mathrm{N}=710, \mathrm{I}^{2}=0 \%$, $\mathrm{Q}=0$ ) and GLP-1 RAs (SMD $=-0.39,95 \% \mathrm{CI}:-0.54$ to $-0.23, \mathrm{p}<$ $0.001 ; 3$ trials, $\mathrm{N}=166, \mathrm{I}^{2}=0 \%, \mathrm{Q}=0$ ).

No significant reduction in glucose levels compared to the placebo condition was observed for topiramate $(\mathrm{SMD}=-0.43$, 95\% CI: -1.00 to $0.14, \mathrm{p}=0.14 ; 6$ trials, $\mathrm{N}=369, \mathrm{I}^{2}=0 \%, \mathrm{Q}=0$ ).

\section{Insulin}

\section{Non-pharmacological interventions}

Two meta-analyses investigated the effect of non-pharmacological interventions on insulin levels ( 9 trials, $N=1,268)$. The mean AMSTAR score was $9.0 \pm 0.0$ and the mean AMSTAR-Plus Content was $4.5 \pm 0.7$.

Multidisciplinary lifestyle/behavioral interventions had a small effect in improving insulin sensitivity ( $\mathrm{SMD}=-0.28,95 \%$ CI: -0.55 to $0.00, \mathrm{p}=0.04 ; 6$ trials, $\mathrm{N}=481, \mathrm{I}^{2}=0 \%, \mathrm{Q}=0$ ). Dietary interventions alone did not outperform the control condition (SMD $=-0.19,95 \% \mathrm{CI}:-0.42$ to $0.04, \mathrm{p}=0.10 ; 11$ trials, $\mathrm{N}=787$, $\left.\mathrm{I}^{2}=0 \%, \mathrm{Q}=0\right)$.

\section{Pharmacological interventions}

Five meta-analyses investigated the impact of pharmacological interventions on insulin levels (23 trials, $\mathrm{N}=1,479$ ). The mean AMSTAR score was $8.4 \pm 1.2$ and the mean AMSTAR-Plus Content was $4.2 \pm 2.2$.

Rosiglitazone had a small effect (SMD $=-0.42,95 \% \mathrm{CI}:-0.80$ to $0.00, \mathrm{p}=0.03$; one trial, $\mathrm{N}=29$ ). Metformin did not outperform the control condition (SMD $=-0.37,95 \% \mathrm{CI}$ : -0.81 to 0.07 , $\mathrm{p}=0.10 ; 15$ trials, $\left.\mathrm{N}=1007, \mathrm{I}^{2}=79.2 \%, \mathrm{Q}=4.8\right)$.

\section{Homeostatic model assessment of insulin resistance}

\section{Pharmacological interventions}

Five meta-analyses investigated the effect of pharmacological interventions on homeostatic model assessment of insulin resistance ( 19 trials, $\mathrm{N}=1,158$ ). The mean AMSTAR score was 8.4 \pm 1.2 and the mean AMSTAR-Plus Content was 4.2 \pm 2.2 . 
Metformin (SMD=-0.75; 95\% CI: -1.10 to $-0.40, \mathrm{p}<0.001 ; 11$ trials, $\mathrm{N}=680, \mathrm{I}^{2}=0 \%, \mathrm{Q}=0$, i.e., medium effect) and rosiglitazone ( $\mathrm{SMD}=-0.44 ; 95 \% \mathrm{CI}$ : -0.82 to $-0.06, \mathrm{p}=0.02$; one trial, $\mathrm{N}=29$, i.e., small effect) significantly outperformed the control condition, while GLP-1 RAs did not (SMD=-0.08; 95\% CI: -0.23 to 0.08 , $\mathrm{p}=0.10 ; 3$ trials, $\left.\mathrm{N}=163, \mathrm{I}^{2}=0 \%, \mathrm{Q}=0\right)$.

\section{Hemoglobin A1c}

Two meta-analyses investigated pharmacological interventions on hemoglobin A1c ( 7 trials, $\mathrm{N}=549$ ). The mean AMSTAR score was $9.0 \pm 0.0$ and the mean AMSTAR-Plus Content was 3.5 \pm 3.5 .

Metformin (SMD $=-0.38 ; 95 \% \mathrm{CI}:-0.69$ to $-0.07, \mathrm{p}=0.016 ; 4$ trials, $\mathrm{N}=383, \mathrm{I}^{2}=0 \%, \mathrm{Q}=0$ ) and GLP-1 RAs ( $\mathrm{SMD}=-0.38 ; 95 \%$ CI: -0.53 to -0.22 , $p=0.02 ; 3$ trials, $N=166, I^{2}=0 \%, Q=0$ ) outperformed the placebo condition and showed a small effect.

\section{Triglycerides}

\section{Non-pharmacological interventions}

Four meta-analyses investigated the impact of non-pharmacological interventions on triglycerides (22 trials, $N=1,671$ ). The mean AMSTAR score was 8.7 \pm 1.1 and the mean AMSTAR-Plus Content was $4.2 \pm 0.8$.

Multidisciplinary lifestyle/behavioral interventions had a small effect compared with care as usual (SMD $=-0.37,95 \% \mathrm{CI}$ : -0.54 to $-0.20, \mathrm{p}<0.001 ; 8$ trials, $\left.\mathrm{N}=659, \mathrm{I}^{2}=40.0 \%, \mathrm{Q}=1.7\right)$. Dietary interventions did not outperform care as usual (SMD= -0.15 ; $95 \%$ CI: -0.30 to $0.00, \mathrm{p}=0.06$; 7 trials, $\mathrm{N}=611, \mathrm{I}^{2}=0 \%, \mathrm{Q}=0$ ).

\section{Pharmacological interventions}

Seven meta-analyses investigated the effect of pharmacological interventions on triglycerides (36 trials, $\mathrm{N}=2,564$ ). The mean AMSTAR score was 9.1 \pm 1.0 and the mean AMSTAR-Plus Content was $3.6 \pm 1.9$.

The effect compared with control condition was medium for topiramate (SMD=-0.68, 95\% CI: -1.23 to -0.13 , $\mathrm{p}=0.016$; 5 trials, $\mathrm{N}=268, \mathrm{I}^{2}=0 \%, \mathrm{Q}=0$ ), small for metformin ( $\mathrm{SMD}=-0.28,95 \%$ CI: -0.45 to $-0.11, \mathrm{p}<0.001 ; 11$ trials, $\mathrm{N}=856, \mathrm{I}^{2}=5 \%, \mathrm{Q}=2.1$ ), and negligible for aripiprazole augmentation (SMD $=-0.17,95 \% \mathrm{CI}$ : -0.30 to $-0.04, \mathrm{p}=0.009 ; 9$ trials, $\left.\mathrm{N}=631, \mathrm{I}^{2}=0 \%, \mathrm{Q}=0\right)$ and GLP-1 RAs (SMD $=-0.15,95 \%$ CI: -0.31 to $-0.02, \mathrm{p}=0.04 ; 3$ trials, $\mathrm{N}=166$, $\left.\mathrm{I}^{2}=0 \%, \mathrm{Q}=0\right)$.

\section{Cholesterol}

\section{Non-pharmacological interventions}

Ten meta-analyses investigated non-pharmacological interventions for cholesterol levels ( 56 trials, $\mathrm{N}=4,288$ ). The mean
AMSTAR score was $8.5 \pm 1.0$ and the mean AMSTAR-Plus Content was $4.5 \pm 0.7$.

Regarding total cholesterol, multidisciplinary lifestyle/behavioral interventions had a small benefit (SMD=-0.35, 95\% CI: -0.54 to $-0.16, \mathrm{p}=0.003 ; 7$ trials, $\mathrm{N}=590, \mathrm{I}^{2}=0 \%, \mathrm{Q}=0.3$ ), while dietary interventions alone did not outperform care as usual (SMD=-0.13; 95\% CI: -0.29 to $0.03, \mathrm{p}=0.10 ; 7$ trials, $\mathrm{N}=621, \mathrm{I}^{2}=0 \%, \mathrm{Q}=0$ ).

Regarding LDL-cholesterol, multidisciplinary lifestyle/behavioral interventions showed a small benefit (SMD=-0.36, 95\% CI: -0.60 to -0.12 , $\mathrm{p}=0.003 ; 5$ trials, $\mathrm{N}=590, \mathrm{I}^{2}=0 \%, \mathrm{Q}=0.2$ ).

No significant effects on HDL-cholesterol elevations were found with lifestyle or dietary interventions.

\section{Pharmacological interventions}

Fifteen meta-analyses investigated pharmacological interventions for cholesterol levels ( 74 trials, $\mathrm{N}=5,295$ ). The mean AMSTAR score was $9.4 \pm 0.8$ and the mean AMSTAR-Plus Content was $3.3 \pm 1.8$.

Regarding total cholesterol, metformin (SMD=-0.51, 95\% CI: -0.81 to $-0.20, \mathrm{p}<0.001 ; 8$ trials, $\mathrm{N}=628, \mathrm{I}^{2}=0 \%, \mathrm{Q}=0$ ) demonstrated a medium effect, while aripiprazole augmentation had a small effect $(\mathrm{SMD}=-0.32,95 \% \mathrm{CI}=-0.47$ to $-0.17, \mathrm{p}<0.001$; 10 trials, $\mathrm{N}=692, \mathrm{I}^{2}=0 \%, \mathrm{Q}=0$ ). No significant reduction compared to the control condition was observed for topiramate (SMD $=-0.75,95 \% \mathrm{CI}:-1.57$ to $0.07, \mathrm{p}=0.07 ; 3$ trials, $\mathrm{N}=187, \mathrm{I}^{2}=0 \%$, $\mathrm{Q}=0)$.

Regarding LDL-cholesterol, topiramate (SMD=-0.80, 95\% CI: -1.06 to $-0.53, \mathrm{p}<0.001 ; 4$ trials, $\left.\mathrm{N}=247, \mathrm{I}^{2}=0 \%, \mathrm{Q}=0\right)$ and GLP-1 RAs (SMD=-0.17, 95\% CI: -0.32 to -0.02 , $\mathrm{p}=0.04 ; 3$ trials, $\mathrm{N}=162, \mathrm{I}^{2}=0 \%, \mathrm{Q}=0$ ) outperformed the control condition, although for the latter the effect was negligible. No significant reductions compared to the control condition were found for aripiprazole augmentation (SMD $=-0.01,95 \% \mathrm{CI}:-0.18$ to $0.15, \mathrm{p}=0.88$; 8 trials, $\mathrm{N}=540, \mathrm{I}^{2}=0 \%, \mathrm{Q}=0$ ) and for metformin $\left(\mathrm{SMD}=-0.11,95 \% \mathrm{CI}:-0.31\right.$ to $0.09, \mathrm{p}=0.29 ; 5$ trials, $\mathrm{N}=433, \mathrm{I}^{2}$ $=0 \%, \mathrm{Q}=0.2$ ).

Regarding HDL-cholesterol, only metformin had a small effect (SMD=0.45, 95\% CI: 0.00 to $0.90, \mathrm{p}=0.049 ; 7$ trials, $\mathrm{N}=542$, $\mathrm{I}^{2}=0 \%, \mathrm{Q}=0$ ), while aripiprazole augmentation, topiramate and GLP-1 RAs did not differ from the control condition.

\section{Functional exercise capacity}

Based on data from one meta-analysis, exercise outperformed the treatment as usual condition (SMD=1.81; 95\% CI: 0.59 to 3.03, $\mathrm{p}=0.004$; one trial, $\mathrm{N}=13, \mathrm{I}^{2}=0 \%, \mathrm{Q}=0$, i.e., large effect).

\section{Adverse drug reactions}

Compared to placebo, aripiprazole had higher rates of anxiety (number needed to harm, $\mathrm{NNH}=8,95 \% \mathrm{CI}$ : 5 to $20, \mathrm{p}<$ $0.001)^{47}$ and agitation/akathisia (RR=7.59, 95\% CI: 1.43 to 40.18 , 
$\mathrm{p}=0.02)^{53}$. Amantadine was associated with higher rates of insomnia ( $R R=3.83,95 \% \mathrm{CI}$ : 1.41 to $10.38, \mathrm{p}=0.008, \mathrm{NNH}=9)^{57}$ and abdominal discomfort (quantitative data not provided) ${ }^{60}$. GLP-1 RAs were associated with higher rates of nausea $(\mathrm{NNH}=$ 3.8, 95\% CI: 2.4 to 9.7, $\mathrm{p}<0.05)^{40}$. Among H2 antagonists, famotidine and ranitidine were not associated with higher rates of adverse reactions, while nizatidine had higher rates of dry mouth $(\mathrm{RR}=4.89, \mathrm{p}=0.04 ; \mathrm{NNH}=17, \mathrm{p}=0.03)$ and depression $(\mathrm{RR}=5.00$, $\mathrm{p}=0.03 ; \mathrm{NNH}=17, \mathrm{p}=0.02)^{52}$.

Of the six included meta-analyses of metformin, five reported no difference in rates of adverse reactions ${ }^{41,46,48,54,62}$, while one reported higher rates of nausea/vomiting $(\mathrm{NNH}=16,95 \%$ CI: 10 to $50, p=0.01$ ) and diarrhoea ( $\mathrm{NNH}=6,95 \% \mathrm{CI}: 3$ to $25, \mathrm{p}=$ $0.01)^{49}$. Although there was no difference in rates of dropouts for orlistat, diarrhoea was the main reason for discontinuation of this drug ${ }^{48}$.

Sibutramine plus metformin were associated with an increase of psychotic symptoms. Topiramate was associated with a higher rate of paraesthesia $(\mathrm{RR}=2.31,95 \% \mathrm{CI}: 1.17$ to $4.56, \mathrm{p}<0.05)^{39}$. There were no data for dextroamphetamine, d-fenfluramine and rosiglitazone. Antipsychotic switching (olanzapine to quetiapine) was associated with higher rates of psychiatric adverse events ${ }^{54}$.

None of the meta-analyses reported on adverse effects of non-pharmacological interventions.

\section{Meta-regression analyses}

Due to limited data, no meta-regression or subgroup analysis could be performed to examine whether duration of treatment or illness, delivery of the intervention by mental health vs. physical health staff, clinical setting, percent of sessions attended or adherence to treatment could explain variance in the outcomes of interventions.

Study level variance in age and gender did not explain the variance in weight, body mass index or triglycerides levels following pharmacological or non-pharmacological interventions.

\section{DISCUSSION}

To our knowledge, this meta-review of meta-analyses is the first to systematically and quantitatively compare the pharmacological and non-pharmacological interventions that have been investigated for improving physical health outcomes in people with schizophrenia. Our data shed new light on the areas where there is or there is not evidence to improve physical health in these people, and should help guide clinical practice and indicate where future research priorities should focus.

When looking at non-pharmacological treatments, individual lifestyle counseling showed a large weight reducing effect. For dietary interventions, the weight reducing effect size was medium, and diastolic blood pressure and glucose level low- ering effects were small. Exercise demonstrated large weight reducing effects and large effects on functional exercise capacity, although the evidence for the latter was limited to one small study.

The characteristics of the lifestyle interventions were examined to provide guidelines for future clinical practice. One key finding was that individualized lifestyle interventions showed large effects for reducing body weight, while only a small effect was observed for group-based approaches. Apparently, the benefits of an individual strategy, such as personal advice and attention, meeting patient-specific needs, and a tailored action plan, surpasses the benefits of group-based sessions, such as interpersonal learning, imitative behavior, recognition of similarities in other group members, group cohesiveness and peer support ${ }^{66}$. Future research should, however, explore whether a combined approach, encompassing group sessions while addressing patient-specific needs with a tailored action plan, would be most efficacious.

Cognitive behavioral interventions focusing on weight loss and psychoeducation demonstrated, respectively, small and medium weight reducing effects.

Across 17 pharmacological strategies, 12 outperformed the control condition on various physical health outcomes. No beneficial effects were found for fluoxetine, ranitidine, orlistat, dextroamphetamine and famotidine for any physical health outcome.

Topiramate showed a large effect on LDL-cholesterol, and a medium effect on weight, body mass index, waist circumference and triglycerides. Metformin demonstrated a medium effect on weight, total cholesterol, fasting glucose levels, and homeostatic model assessment of insulin resistance; and a small effect on hemoglobin Alc, triglycerides, and HDL-cholesterol.

Switching from olanzapine to quetiapine or aripiprazole showed a medium fasting glucose lowering effect, while the effect of aripiprazole augmentation on this parameter was small. Aripiprazole augmentation also had a large effect on waist circumference and a medium effect on body weight.

A small weight reducing effect was found for NMDA receptor antagonists. GLP-1 RAs showed small effects on waist circumference, glucose and hemoglobin Alc. Rosiglitazone had a small improving effect on homeostatic model assessment of insulin resistance. Finally, negligible effects were observed for aripiprazole augmentation and GLP-1 RAs on triglycerides, and for GLP-1 RAs on LDL-cholesterol.

In summary, based on the SMDs and the overall high methodological quality of the original meta-analyses (but with lower quality of the meta-analyzed content), individual lifestyle counseling and exercise interventions showed the largest weight reducing effect, followed by psychoeducation, aripiprazole augmentation, topiramate, di-fenfluramine and metformin. With regard to waist circumference, aripiprazole augmentation and topiramate demonstrated the best impact, followed by dietary interventions. Dietary interventions were the only to significantly improve (diastolic) blood pressure. 
Switching from olanzapine to quetiapine or aripiprazole and metformin demonstrated the best evidence for glucose level reductions, followed by GLP-1 RAs, dietary interventions and aripiprazole augmentation. Hemoglobin Alc was reduced significantly by both metformin and GLP-1 RAs. Homeostatic model assessment of insulin resistance improved significantly with metformin and rosiglitazone.

Reduction of triglycerides levels were the largest with topiramate, followed by multidisciplinary lifestyle/behavioral interventions, metformin, aripiprazole augmentation and GLP-1 RAs. Total cholesterol was reduced by metformin, lifestyle interventions and aripiprazole augmentation, while LDLcholesterol reductions were significant with topiramate, lifestyle interventions and GLP-1 RAs. HDL-cholesterol only increased significantly with metformin. Finally, only exercise interventions were meta-analyzed as a means to improve exercise capacity, yielding the largest effect size of all interventions in this review for any outcome (SMD=1.81), although this was based on only one trial.

Taken together, our data offer clinicians some perspective on the potential best methods to address specific physical health issues in people with schizophrenia. In summary, for weight reduction, clinicians should consider individual lifestyle counselling as the top non-pharmacological intervention. There is some evidence that exercise interventions can also help reduce body weight, although we could only include four trials. Dietary interventions also showed promise. Regarding pharmacological interventions, clinicians could consider the adjunctive use of topiramate, though this should be balanced against the possible emergence of paresthesia and cognitive adverse effects (the latter insufficiently studied). Findings for metformin were somewhat heterogeneous, as this medication had a medium effect on body weight but no effect on body mass index, although the latter was likely due to the smaller number of studies examining this outcome. Metformin may be associated with nausea and diarrhea. Further research is required to determine the effects of combining these strategies.

With regards to other markers of metabolic health and cardiovascular risk, there is good evidence that clinicians can use metformin for reducing glucose levels, homeostatic model assessment of insulin resistance and total cholesterol, while there is only a small effect for triglycerides, hemoglobin Alc and HDL-cholesterol. For people with schizophrenia on olanzapine, switching to aripiprazole or quetiapine also shows medium glucose level lowering effects. Of note, only dietary interventions were found to significantly improve (diastolic) blood pressure.

Our data should be considered in the light of some limitations. First, although the included meta-analyses were the most updated and/or largest for each specific strategy and outcome, individual studies published since the last search date of included meta-analyses could not be added. Second, because of limited data for participant characteristics and interventional designs, conducting meta-regression analyses was not possible. Third, while the quality of the methods of the meta-analyses was generally good to very good, the content of meta-analyzed studies often lacked quality. Fourth, based on the AMSTAR-Plus Content scores, publication bias was problematic for about half of the meta-analyses, potentially overestimating the pooled effect sizes. Finally, the preponderance of studies with small sample sizes in which only large effects were statistically significant presents a challenge.

In conclusion, despite the high risk for physical comorbidities in people with schizophrenia, and the scandal of their premature mortality mainly due to these increased physical health risks, the existing evidence for pharmacological and non-pharmacological interventions to prevent and treat these conditions is still limited. Qualitatively excellent and sufficiently large individual randomized clinical trials are therefore essential.

Additionally, the field should move from study-level to patient-level meta-analyses, as this would provide a more personalized picture of treatment effects for individuals, derived from adequately powered moderator, mediator and subgroup analyses. Comparing pharmacological and non-pharmacological interventions in the same trial would also be desirable, and there is a need for large-scale investigations of combination regimes (i.e., using antipsychotic switching and adjunctive prescribing alongside lifestyle interventions), as well as preventive interventions (i.e., those aiming to prevent physical comorbidities, prior to their development).

\section{ACKNOWLEDGEMENTS}

B. Stubbs holds a clinical lectureship supported by Health Education England and the National Institute for Health Research (NIHR) Integrated Clinical Academic Programme (ICA-CL-2017-03-001). B. Stubbs and F. Gaughran are in part supported by the Maudsley Charity and the NIHR Collaboration for Leadership in Applied Health Research and Care South London at King's College Hospital NHS Foundation Trust. The views expressed in this paper are those of the authors and not necessarily those of the above entities.

\section{REFERENCES}

1. Vancampfort D, Wampers M, Mitchell A et al. A meta-analysis of cardiometabolic abnormalities in drug naïve, first-episode and multi-episode patients with schizophrenia versus general population controls. World Psychiatry 2013;12:240-50.

2. Stubbs B, De Hert M, Sepehry A et al. A meta-analysis of prevalence estimates and moderators of low bone mass in people with schizophrenia. Acta Psychiatr Scand 2014;130:470-86.

3. Stubbs B, Koyanagi A, Veronese $\mathrm{N}$ et al. Physical multimorbidity and psychosis: comprehensive cross sectional analysis including 242,952 people across 48 low- and middle-income countries. BMC Med 2016;14:189.

4. Vancampfort D, Stubbs B, Mitchell AJ et al. Risk of metabolic syndrome and its components in people with schizophrenia and related psychotic disorders, bipolar disorder and major depressive disorder: a systematic review and meta-analysis. World Psychiatry 2015;14:339-47.

5. Liu NH, Daumit GL, Dua T et al. Excess mortality in persons with severe mental disorders: a multilevel intervention framework and priorities for clinical practice, policy and research agendas. World Psychiatry 2017;16: 30-40.

6. Vancampfort D, Firth J, Schuch FB et al. Sedentary behavior and physical activity levels in people with schizophrenia, bipolar disorder and major depressive disorder: a global systematic review and meta-analysis. World Psychiatry 2017;16:308-15.

7. Firth J, Stubbs B, Teasdale SB et al. Diet as a hot topic in psychiatry: a population-scale study of nutritional intake and inflammatory potential in severe mental illness. World Psychiatry 2018;17:365-6. 
8. Schroeder SA. Smoking cessation should be an integral part of serious mental illness treatment. World Psychiatry 2016;15:175-6.

9. Correll C, Detraux J, De Lepeleire J et al. Effects of antipsychotics, antidepressants and mood stabilizers on risk for physical diseases in people with schizophrenia, depression and bipolar disorder. World Psychiatry 2015;14:119-36.

10. Galling B, Roldán A, Nielsen RE et al. Type 2 diabetes mellitus in youth exposed to antipsychotics: a systematic review and meta-analysis. JAMA Psychiatry 2016;73:247-59.

11. Vancampfort D, Correll CU, Galling B et al. Diabetes mellitus in people with schizophrenia, bipolar disorder and major depressive disorder: a systematic review and large scale meta-analysis. World Psychiatry 2016;15:166-74.

12. De Hert M, Schreurs V, Vancampfort D et al. Metabolic syndrome in people with schizophrenia: a review. World Psychiatry 2009;8:15-22.

13. Correll CU, Solmi M, Veronese $\mathrm{N}$ et al. Prevalence, incidence and mortality from cardiovascular disease in patients with pooled and specific severe mental illness: a large-scale meta-analysis of 3,211,768 patients and 113,383,368 controls. World Psychiatry 2017;16:163-80.

14. Schoepf D, Uppal H, Potluri R et al. Physical comorbidity and its relevance on mortality in schizophrenia: a naturalistic 12-year follow-up in general hospital admissions. Eur Arch Clin Neurosci 2014;264:3-28.

15. Stubbs B, Koyanagi A, Thompson $\mathrm{T}$ et al. The epidemiology of back pain and its relationship with depression, psychosis, anxiety, sleep disturbances, and stress sensitivity: data from 43 low- and middle-income countries. Gen Hosp Psychiatry 2016;43:63-70.

16. Stubbs B, Gaughran F, Mitchell AJ et al. Schizophrenia and the risk of fractures: a systematic review and comparative meta-analysis. Gen Hosp Psychiatry 2015;37:126-33.

17. Vancampfort D, Rosenbaum S, Probst M et al. Promotion of cardiorespiratory fitness in schizophrenia: a clinical overview and meta-analysis. Acta Psychiatr Scand 2015;132:131-43.

18. Vancampfort D, Rosenbaum S, Schuch F et al. Cardiorespiratory fitness in severe mental illness: a systematic review and meta-analysis. Sports Med 2017;47:343-52.

19. Vancampfort D, Guelinckx H, Probst M et al. Health-related quality of life and aerobic fitness in people with schizophrenia. Int J Ment Health Nurs 2015;24:394-402.

20. Vancampfort D, Probst M, Scheewe T et al. Lack of physical activity during leisure time contributes to an impaired health related quality of life in patients with schizophrenia. Schizophr Res 2011;129:122-7.

21. Saxena S, Maj M. Physical health of people with severe mental disorders: leave no one behind. World Psychiatry 2017;16:1-2.

22. Mitchell AJ, Delaffon V, Vancampfort D et al. Guideline concordant monitoring of metabolic risk in people treated with antipsychotic medication: systematic review and meta-analysis of screening practices. Psychol Med 2012;42:125-47.

23. Zhuo C, Tao R, Jiang R et al. Cancer mortality in patients with schizophrenia: systematic review and meta-analysis. Br J Psychiatry 2017;211:7-13.

24. Hjorthøj C, Stürup AE, McGrath JJ et al. Years of potential life lost and life expectancy in schizophrenia: a systematic review and meta-analysis. Lancet Psychiatry 2017;4:295-301.

25. Oakley P, Kisely S, Baxter A et al. Increased mortality among people with schizophrenia and other non-affective psychotic disorders in the community: a systematic review and meta-analysis. J Psychiatr Res 2018;102:245-53.

26. Moore S, Shiers D, Daly B et al. Promoting physical health for people with schizophrenia by reducing disparities in medical and dental care. Acta Psychiatr Scand 2015;132:109-21.

27. De Hert M, Cohen D, Bobes J et al. Physical illness in patients with severe mental disorders. II. Barriers to care, monitoring and treatment guidelines, plus recommendations at the system and individual level. World Psychiatry 2011;10:138-51.

28. Lambert TJ, Reavley NJ, Jorm AF et al. Royal Australian and New Zealand College of Psychiatrists expert consensus statement for the treatment, management and monitoring of the physical health of people with an enduring psychotic illness. Aust N Z J Psychiatry 2017;51:322-37.

29. Cooper SJ, Reynolds GP, Barnes T et al. BAP guidelines on the management of weight gain, metabolic disturbances and cardiovascular risk associated with psychosis and antipsychotic drug treatment. J Psychopharmacol 2016;30: 717-48.

30. Bobes-García J, Saiz-Ruiz J, Bernardo-Arroyo M et al. Delphi consensus on the physical health of patients with schizophrenia: evaluation of the recommendations of the Spanish Societies of Psychiatry and Biological Psychiatry by a panel of experts. Actas Esp Psiquiatr 2012;40:114-28.
31. Chaudhry I, Jordan J, Cousin F-R et al. Management of physical health in patients with schizophrenia: international insights. Eur Psychiatry 2010;25:S37-40.

32. Saravane D, Feve B, Frances Y et al. Drawing up guidelines for the attendance of physical health of patients with severe mental illness. Encephale 2009;35:330-9.

33. De Hert M, Dekker JM, Wood D et al. Cardiovascular disease and diabetes in people with severe mental illness position statement from the European Psychiatric Association (EPA), supported by the European Association for the Study of Diabetes (EASD) and the European Society of Cardiology (ESC). Eur Psychiatry 2009;24:412-24

34. Marder SR, Essock SM, Miller AL et al. Physical health monitoring of patients with schizophrenia. Am J Psychiatry 2004;161:1334-49.

35. Cohen J. Statistical power analysis for the behavioral sciences. New York: Lawrence Erlbaum, 1988.

36. Shea BJ, Hamel C, Wells GA et al. AMSTAR is a reliable and valid measurement tool to assess the methodological quality of systematic reviews. J Clin Epidemiol 2009;62:1013-20.

37. Correll CU, Rubio JM, Inczedy-Farkas G et al. Efficacy of 42 pharmacologic cotreatment strategies added to antipsychotic monotherapy in schizophrenia: systematic overview and quality appraisal of the meta-analytic evidence. JAMA Psychiatry 2017;74:675-84.

38. Higgins JPT, Thompson SG, Deeks JJ et al. Measuring inconsistency in metaanalyses. BMJ 2003;327:557-60.

39. Goh KK, Chen C-H, Lu M-L. Topiramate mitigates weight gain in antipsychotic-treated patients with schizophrenia: meta-analysis of randomised controlled trials. Int J Psychiatry Clin Pract 2018:1-19.

40. Siskind D, Hahn M, Correll CU et al. Glucagon-like peptide-1 receptor-agonists for antipsychotic-associated cardio-metabolic risk factors: a systematic review and individual participant data meta-analysis. Diabetes Obes Metab (in press).

41. Zheng W, Zhang Q-E, Cai D-B et al. Combination of metformin and lifestyle intervention for antipsychotic-related weight gain: a meta-analysis of randomized controlled trials. Pharmacopsychiatry (in press).

42. Gu X-J, Chen R, Sun C-H et al. Effect of adjunctive ranitidine for antipsychotic-induced weight gain: a systematic review of randomized placebocontrolled trials. J Int Med Res 2018;46:22-32.

43. Galling B, Roldán A, Hagi K et al. Antipsychotic augmentation vs. monotherapy in schizophrenia: systematic review, meta-analysis and meta-regression analysis. World Psychiatry 2017;16:77-89.

44. Teasdale SB, Ward PB, Rosenbaum S et al. Solving a weighty problem: systematic review and meta-analysis of nutrition interventions in severe mental illness. Br J Psychiatry 2017;210:110-8.

45. Zheng W, Wang S, Ungvari GS et al. Amantadine for antipsychotic-related weight gain: meta-analysis of randomized placebo-controlled trials. J Clin Psychopharmacol 2017;37:341-6.

46. Siskind DJ, Leung J, Russell AW et al. Metformin for clozapine associated obesity: a systematic review and meta-analysis. PLoS One 2016;11:e0156208.

47. Zheng W, Zheng Y-J, Li X-B et al. Efficacy and safety of adjunctive aripiprazole in schizophrenia: meta-analysis of randomized controlled trials. J Clin Psychopharmacol 2016;36:628-36.

48. Zimbron J, Khandaker GM, Toschi C et al. A systematic review and meta-analysis of randomised controlled trials of treatments for clozapineinduced obesity and metabolic syndrome. Eur Neuropsychopharmacol 2016;26:1353-65.

49. Zheng W, Li X-B, Tang Y-L et al. Metformin for weight gain and metabolic abnormalities associated with antipsychotic treatment: meta-analysis of randomized placebo-controlled trials. J Clin Psychopharmacol 2015;35:499-509.

50. Firth J, Cotter J, Elliott R et al. A systematic review and meta-analysis of exercise interventions in schizophrenia patients. Psychol Med 2015;45:1343-61.

51. Bruins J, Jörg F, Bruggeman R et al. The effects of lifestyle interventions on (long-term) weight management, cardiometabolic risk and depressive symptoms in people with psychotic disorders: a meta-analysis. PLoS One 2014; 9:e112276.

52. Kishi T, Iwata N. Efficacy and tolerability of histamine-2 receptor antagonist adjunction of antipsychotic treatment in schizophrenia: a meta-analysis of randomized placebo-controlled trials. Pharmacopsychiatry 2015;48:30-6.

53. Srisurapanont $M$, Suttajit S, Maneeton $N$ et al. Efficacy and safety of aripiprazole augmentation of clozapine in schizophrenia: a systematic review and meta-analysis of randomized-controlled trials. J Psychiatr Res 2015;62:38-47.

54. Gierisch JM, Nieuwsma JA, Bradford DW et al. Pharmacologic and behavioral interventions to improve cardiovascular risk factors in adults with serious mental illness: a systematic review and meta-analysis. J Clin Psychiatry 2014;75:e424-40. 
55. Mizuno Y, Suzuki T, Nakagawa A et al. Pharmacological strategies to counteract antipsychotic-induced weight gain and metabolic adverse effects in schizophrenia: a systematic review and meta-analysis. Schizophr Bull 2014; 40:1385-403.

56. Pearsall R, Smith DJ, Pelosi A et al. Exercise therapy in adults with serious mental illness: a systematic review and meta-analysis. BMC Psychiatry 2014; 14:117.

57. Kishi T, Iwata N. NMDA receptor antagonists interventions in schizophrenia: meta-analysis of randomized, placebo-controlled trials. J Psychiatr Res 2013;47:1143-9.

58. Bonfioli E, Berti L, Goss C et al. Health promotion lifestyle interventions for weight management in psychosis: a systematic review and meta-analysis of randomised controlled trials. BMC Psychiatry 2012;12:78.

59. Caemmerer J, Correll CU, Maayan L. Acute and maintenance effects of nonpharmacologic interventions for antipsychotic associated weight gain and metabolic abnormalities: a meta-analytic comparison of randomized controlled trials. Schizophr Res 2012;140:159-68.

60. Praharaj SK, Sharma PSVN. Amantadine for olanzapine-induced weight gain: a systematic review and meta-analysis of randomized placebo-controlled trials. Ther Adv Psychopharmacol 2012;2:151-6.
61. Björkhem-Bergman L, Asplund AB, Lindh JD. Metformin for weight reduction in non-diabetic patients on antipsychotic drugs: a systematic review and meta-analysis. J Psychopharmacol 2011;25:299-305.

62. Praharaj SK, Jana AK, Goyal N et al. Metformin for olanzapine-induced weight gain: a systematic review and meta-analysis. Br J Clin Pharmacol 2011;71:377-82.

63. Alvarez-Jimenez M, Hetrick SE, Gonzalez-Blanch C et al. Non-pharmacological management of antipsychotic-induced weight gain: systematic review and meta-analysis of randomised controlled trials. Br J Psychiatry 2008;193:101-7.

64. Maayan L, Vakhrusheva J, Correll CU. Effectiveness of medications used to attenuate antipsychotic-related weight gain and metabolic abnormalities: a systematic review and meta-analysis. Neuropsychopharmacology 2010;35:1520-30.

65. Mukundan A, Faulkner G, Cohn T et al. Antipsychotic switching for people with schizophrenia who have neuroleptic-induced weight or metabolic problems. Cochrane Database Syst Rev 2010;12:CD006629.

66. Yalom ID. Inpatient group psychotherapy. New York: Basic Books, 1983.

DOI:10.1002/wps.20614 